\title{
BMJ Open Paramedics' experiences of administering fascia iliaca compartment block to patients in South Wales with suspected hip fracture at the scene of injury: results of focus groups
}

Bridie Angela Evans, ${ }^{1}$ Alan Brown, ${ }^{2}$ Jenna Bulger, ${ }^{1}$ Greg Fegan, ${ }^{1}$ Simon Ford, ${ }^{3}$ Katy Guy, ${ }^{4}$ Slan Jones, ${ }^{2}$ Leigh Keen, ${ }^{5}$ Ashrafunnesa Khanom, ${ }^{1}$ Mirella Longo, ${ }^{6}$ lan Pallister, ${ }^{3}$ Nigel Rees, ${ }^{7}$ Ian T Russell, ${ }^{1}$ Anne C Seagrove, ${ }^{1}$ Alan Watkins, ${ }^{1}$ Helen Snooks ${ }^{\oplus 1}$

To cite: Evans BA, Brown A, Bulger J, et al. Paramedics' experiences of administering fascia iliaca compartment block to patients in South Wales with suspected hip fracture at the scene of injury: results of focus groups. BMJ Open 2019;9:e026073. doi:10.1136/ bmjopen-2018-026073

\section{- Prepublication history and} additional material for this paper are available online. To view these files, please visit the journal online (http://dx.doi. org/10.1136/bmjopen-2018026073).

Received 17 August 2018 Revised 20 December 2018 Accepted 27 December 2018

Check for updates

(C) Author(s) (or their employer(s)) 2019. Re-use permitted under CC BY-NC. No commercial re-use. See rights and permissions. Published by BMJ.

For numbered affiliations see end of article.

Correspondence to

Bridie Angela Evans;

b.a.evans@swansea.ac.uk

\section{ABSTRACT}

Objectives To explore paramedics' experience of delivering fascia iliaca compartment block (FICB) to patients with suspected hip fracture at the scene of injury.

Design Focus groups within a randomised controlled trial. Setting Paramedics based at ambulance stations in the catchment area of one Emergency Department in South Wales, recruited and trained in a feasibility study about an alternative to routine prehospital pain management for patients with suspected hip fracture.

Participants 11 paramedics.

Intervention Paramedic-administered FICB to patients with suspected hip fracture. We randomly allocated eligible patients to FICB, a local anaesthetic injection directly into the hip region-or usual care, most commonly morphine using audited scratch cards.

Outcomes Paramedics' experiences of administering FICB gathered through thematic analysis of interview transcripts by two researchers, one paramedic and one lay member.

Results Respondents believed that FICB was a suitable intervention for paramedics to deliver. It aligned with routine practice and was within people's capabilities. They said it took up to 10 minutes longer than usual care to prepare and deliver, in part due to nervousness and unfamiliarity with a new procedure. They praised the training provided but said they were anxious about causing harm by injecting into the wrong location. Confidence increased after one paramedic team successfully treated a patient for local anaesthetic toxicity. Reported challenges related to the emergency context: patients often waited many hours for ambulance arrival; moving patients exacerbated their pain; family and neighbours were present as paramedics administered treatment.

Conclusions Paramedics are willing and able to administer FICB to patients with suspected hip fracture before ambulance transport to hospital. Feasibility study findings will inform further research.

Trial registration number ISRCTN60065373; Pre results.

\section{Strengths and limitations of this study}

- This study is the first to report paramedics' experience of learning and administering fascia iliaca compartment block (FICB) in the prehospital setting.

- Focus group data provide a rare insight into how paramedics view a potentially extended and more clinically skilled role for their profession.

- Respondents' views may not be typical of all paramedics, including trial paramedics who did not participate in focus groups, or those not in the study.

- Paramedics expressed frustration at the small number of FICBs they administered, which was due to the feasibility study design.

- Understanding paramedics' ability and experiences of administering FICB will inform delivery of a future fully powered randomised controlled trial.

\section{BACKGROUND}

Paramedics are continually widening their role as their repertoire of skills, procedures and treatments changes and grows. The scope and range of emergency treatment has evolved since development of the first paramedic programmes, in order to benefit patients and improve efficiency and effectiveness of health services. ${ }^{1}$ Emergency ambulances are increasingly seen as mobile treatment centres while the paramedic role has become more clinically skilled. ${ }^{2}$ Although variable by country and region, paramedic scope of practice goes beyond lifesaving and emergency care. ${ }^{3}$

Hip fracture is predicted to rise to 6.3 million incidents a year worldwide by $2050^{4}$. In the UK, hip fractures result in more admissions to orthopaedic trauma wards than patients with any other injury, ${ }^{5}$ representing 
an annual financial burden of approximately $£ 2$ billion to the National Health Service (NHS). ${ }^{6}$ The injury can be devastating to patients. Delays to surgery (over 48 hours) are associated with postoperative pneumonia and pressure sores. There is a high mortality rate $\left(30 \%\right.$ at 1 year). ${ }^{7}$

Current prehospital pain relief for patients with hip fracture is poor ${ }^{8}$ and may be detrimental to patients in the long term. ${ }^{9}$ Intravenous opiates (usually morphine) are most commonly given by paramedics, ${ }^{8}$ but can be relatively ineffective for dynamic pain which a patient is likely to experience during movement to the ambulance and conveyance to hospital. ${ }^{9}$ Importantly, opiates can cause numerous serious side effects, including nausea, constipation, delirium and respiratory depression. These may delay surgery, require further treatment and worsen patient outcomes. ${ }^{10}$ Additionally, patients report inadequate pain relief for this severely painful injury with up to $40 \%$ of patients not receiving any pain relief. ${ }^{11}$

Fascia iliaca compartment block (FICB) - a local anaesthetic injection directly into the hip region-is routinely used in the emergency department (ED) by medical and increasingly, nurse practitioners, for pain relief. Although this procedure may provide effective analgesia in the prehospital setting ${ }^{12}$ as well as allow the reduction of morphine administration, it is not known whether it would improve patient outcomes or be cost effective. We conducted a study to assess the feasibility of undertaking a fully powered multicentre pragmatic randomised trial to test the clinical and cost effectiveness of paramedics providing FICB as early pain relief at the scene of their injuries for patients who have fractured their hip. ${ }^{13}$ As part of this feasibility study, we explored paramedics' experiences of administering FICB to patients with suspected hip fracture. Appropriate and well-conducted qualitative research can make an important contribution to feasibility studies for randomised controlled trials providing information on acceptability and practical implementation issues. ${ }^{1415}$

In this paper, we report the views and experiences of study paramedics who were trained to administer FICB before hospital admission and who attended patients needing emergency treatment for suspected hip fracture.

\section{METHODS \\ Setting}

We carried out a feasibility trial of paramedic administered FICB for suspected hip fracture, the RAPID trial (rapid analgesia for prehospital hip disruption). Full details are available in our published protocol. ${ }^{16}$ We recruited and trained 19 paramedics based at ambulance stations in the catchment area of one ED in South Wales. They used scratch cards to randomly allocate eligible patients with suspected hip fracture to receive FICB or normal care. ${ }^{17}$

\section{Intervention}

We trained paramedics through an online package including a video showing administration of FICB, followed by group sessions led by a consultant anaesthetist (SF). We used the landmark technique to locate correct administration of the anaesthetic ${ }^{18}{ }^{19}$ since this built on paramedics' existing skills relating to palpation of pulses, bony landmarks and use of needles and is equivalent to the method used in EDs. Paramedics received information about drug dosage, administration technique and risks, reviewed equipment and use of the drug intralipid to reverse local anaesthetic toxicity to FICB according to a predefined protocol. ${ }^{16}$ Pairs of paramedics then attended sessions at the participating hospital where they administered FICB to awake patients observed by an anaesthetist, alternating between administering and critiquing their partner to ensure active learning. ${ }^{20}$ They passed a competency assessment before recruiting patients to the study. They administered FICB to 17 eligible patients during the study. ${ }^{13}$ They attended refresher training and had access to the paramedic research support officer (LK) for additional training or support. We provided a printed treatment protocol as an aide memoire during recruitment to identify patients meeting inclusion criteria to receive FICB.

\section{Data collection and analysis}

To explore paramedics' experiences of administering FICB, we conducted three focus groups towards the end of patient recruitment. We held focus groups at a local ambulance station. LK contacted all study paramedics to take part by phone or social media and offered an honorarium to encourage attendance. We provided information about the aim of the RAPID trial. Focus groups were led by BAE or JB with JB or AK observing and taking notes. All are experienced qualitative researchers. The focus group schedule is available as online supplementary appendix 1 . With participants' consent, we audio-recorded and transcribed discussions. Focus groups lasted between 30 and $90 \mathrm{~min}$.

We carried out thematic analysis. The analysis team included a lay member (SJ), paramedic research support officer (LK) and two researchers (BAE and JB). They independently read transcripts and made notes before jointly discussing explicit and implicit ideas to develop themes. We looked for consistency between respondents and diverse views also. BAE coordinated discussions and prepared drafts, for critical review by the study team. ${ }^{21}$

\section{Reporting}

We report results according to themes identified in the data. We selected quotations to be representative of respondents' comments unless otherwise stated. Quotations from focus groups are identified by FG01, FG02 or FG03, then a number identifying the respondent paramedic (eg, FG02-6).

\section{Patient and public involvement}

Lay members ( $\mathrm{SJ}$ and $\mathrm{AB}$ ) with experience and knowledge of hip fractures and emergency care contributed to developing, undertaking and disseminating all aspects 
of the research. We supported them to collaborate as equal members of the research team throughout. ${ }^{22}$ They were involved in developing the study as coapplicants, using personal experience to highlight relevance of the research questions and comment on data collection methods and selection of outcomes. They prepared participant information sheets. SJ coanalysed qualitative data. They both contributed to study reporting and dissemination through papers, conference presentations and a lay summary.

\section{RESULTS}

Eleven paramedics took part in the three focus groups (FG01-respondents 1-5; FG02-respondents 6-8; FG03-respondents 9-11).

We identified four themes relating to views and experiences of paramedics administering FICB which were consistent across respondents.

\section{Ability and acceptability}

Respondents said they believed that FICB was a suitable intervention for paramedics to administer to treat suspected hip fractures, a regular part of their workload which all were familiar with recognising and treating. They said the process of assessing and communicating with the patient, then administering FICB, fitted into their normal procedures and provided an additional approach to managing a patient's pain. Respondents believed that their ability to work in challenging and urgent situations was exactly the skill which enabled them to incorporate FICB into their working practice.

We're quite used to approaching difficult situations and asking people if we can do something which is probably gonna be a bit uncomfortable or a bit awkward so it's not as if that's anything new. (FG01-3)

Respondents said they experienced a mixture of excitement and nervousness when administering the injection. Although confident about the process for giving the block, their emotions were tempered by anxiety associated with using new equipment and techniques. While it was 'enjoyable and something different' (FG02-6) and 'perks your interest' (FG02-8), they also admitted it adds a little bit of pressure' (FG02-7), was 'nerve wracking' (FG01-3) and 'scary' (FG01-5) to administer the procedure. Most respondents completed training many weeks or months before administering FICB on scene and feared their skills would deteriorate. During the trial, most respondents said they administered the block once or a very few times, so the novelty factor and nerves remained.

Paramedics reported that the challenges of delivering the block arose mainly from the emergency context in which they delivered care. They often found patients in awkward and undignified positions, trapped in narrow corners of bathrooms or bedrooms or having fallen in the garden. Many patients had waited for help for some hours, they said. Respondents worried about exacerbating patients' pain by moving them in order to administer the injection. When family or the public were present, the stress of assessing the emergency, managing the patient and onlookers while also overriding the anxiety of safely injecting the block, created a challenging situation. No patients or family members were reported to have resisted or refused the intervention when offered.

\section{Patient safety and experience}

Paramedics welcomed the block because it provided an alternative pain relief which they believed could be better for patients in the long term, even though many were uncertain whether FICB successfully reduced pain for patients they were treating. However, respondents said the drug was potentially better for patients because it reduced the risk of complications from morphine.

I think it's a fantastic idea to have pre-hospitally, because people die from breaking their hip-they're inactive in hospital, they're pumped full of morphine ... and then they catch a chest infection, and they die. It's something that we can do pre-hospitally, to relieve their pain, but also for them to have a more successful outcome. (FG03-9)

Paramedics reported that having access to the block did not change their approach to caring for patients but some felt it may have increased the time by up to $10 \mathrm{~min}$ before patients received pain relief because respondents took more time to prepare and deliver the intervention.

While paramedics agreed that the FICB procedure was straightforward, they were anxious about patients' response: how pain levels would reduce; causing harm to patients by injecting in the wrong location; or a patient experiencing local anaesthetic toxicity. Patients' reaction became more important to them after one paramedic team had to treat a patient for anaesthetic toxicity. Respondents recognised that patients relied on a paramedic's skills and experience and that they had to be self-reliant when managing urgent and emergency needs.

When you're not feeling the risk, you're not worried. When it's down to you, and you're gonna do something, you need to make sure that you know how to get out of it. (FG03-09)

Although the block was delivered into the patient's groin, respondents did not believe patients felt embarrassed about being handled or exposed in this area of their body. They believed paramedics had good communication skills and could reassure an anxious patient. All judged that patients in acute pain would expect and accept treatment offered by a paramedic.

She'd been on the floor for six hours-I don't think she cared. She said 'give me some pain relief'. (FG02-7) 


\section{Training}

Respondents praised the training, complimenting the trainers and the back-up support provided. They welcomed the mix of classroom and practical sessions including the chance to practice with clinical specialists present and using a training dummy. Several said they kept up their skills by practising block administration on the dummy kept in station. They valued the refresher sessions provided after one patient experienced local anaesthetic toxicity. They suggested ways to improve the training including more prehospital scenario-based training and frequent practice sessions with hospital patients to give them hands-on practice and also refresh their skills for the prehospital setting where conditions were more challenging than in hospital.

Respondents also asked for more experience of opening the packs and being familiar with the contents. When they experienced long gaps between training and administering FICB, they realised their memories had faded and their anxiety rose. Some said they phoned colleagues for reassurance and to confirm what to do. The paramedics who had treated a patient who reacted to the drugs said the real experience was different from the training scenario because the equipment was not what they expected. They recommended specific training for this event.

When somebody was actually deteriorating quite quickly in front of us ... during the heat of the moment there is a lot of things in the pack, a lot of things in the box, two information cards and the potential for confusion is big ... people need to be familiar with this. (FG02-8)

\section{Scope of the paramedic role}

Paramedics in these focus groups welcomed the chance to increase their skills and potentially improve patient care. They talked with interest and enthusiasm about the potential risks and benefits of different medications and management for patients with suspected hip fracture. They also identified how the research potentially contributed towards widening the scope of paramedic practice by extending their role into clinically specialist areas. A paramedic summed up his and colleagues' multiple reasons for signing up with the trial.

One is for the patients, better control of their pain, less use of morphines, which is great. And from a personal point of view, it's personal development, paramedics looking to develop the role and doing surgical procedures ... it's good for the profession and good for us all, as a team, as a group and individually, it's a great opportunity. (FG02-8)

Respondents were keen to celebrate the fact that they were extending their practice and expertise. They described the satisfaction of doing 'extra responsibilities ... developing beyond where we are at the moment' (FG01-5). Another highlighted the status it conferred on paramedicine by using a technique normally reserved for medical staff.

This is quite specialised, like, hang on, I'm doing a really top job here, dealing with anaesthetics ... blunt needles ... this is a specialised area. It's like 'up there'. It's not our everyday thing. (FG01-2)

Many said they were proud to be using new skills and saw themselves as pioneers for the profession. No paramedic colleagues not involved in the study were reported to have resisted administration of the FICB intervention. The one area of frustration concerned the high number of intervention-group patients excluded from receiving FICB because of contraindications, most frequently the use of anticoagulants. It meant that paramedics generally administered just one or two blocks despite attending more patients randomly allocated to receive FICB.

\section{DISCUSSION}

\section{Summary of findings}

Paramedics in this feasibility trial gained skills in a new procedure, successfully administered FICB to patients with suspected hip fracture and successfully reversed one incidence of local anaesthetic toxicity. Although uncertain whether it improved patient experience, they believed it was safer than morphine because it lessened side effects and could improve patient outcomes in the longer term. They welcomed the opportunity to extend their skills by learning a specialist technique and considered they were in the vanguard of paramedicine development.

\section{Implications for practice}

Health services and particularly EDs face growing pressures as demand for services continues to rise and more patients are treated across all disciplines and care levels. ${ }^{23}$ Within approaches to managing demand and speeding patient throughput, healthcare policies in the UK and internationally aim to widen the scope of paramedic practice. ${ }^{1}$ Much focus is on empowering paramedics to treat and refer patients in order to avoid conveyance to hospital, reducing demand in the ED and ensuring those in clinical need have access to transport to ED. ${ }^{24-26}$ This study addresses another driver for enhanced paramedic practice-to improve patient experience before ED arrival, possibly speeding their access to surgery and reducing time before discharge. This potentially improves patient outcomes, reduces heath care costs and frees resources for other patients. ${ }^{13}$ FICB is a safe and effective alternative to opiates. Hospital-administered FICB for patients with hip fracture is reported to reduce length of hospital stay $^{27}$ and may improve patient outcomes. ${ }^{28}{ }^{29}$ Prehospital administration of FICB by nonmedically trained health professionals is supported and feasible. ${ }^{12}{ }^{19}$ This feasibility trial confirms those findings and indicates that FICB can be implemented by nonphysician practitioners in nonhospital settings. It is the first to report paramedics' experiences of learning and delivering FICB. Additionally, it 
provides a rare insight into how paramedics view a potentially extended and more clinically skilled role for their profession. These data demonstrate that paramedics welcome the chance to learn more specialist and clinically focused interventions in order to improve patient care. They perceive that paramedics and patients gain equal benefit from this enhanced approach.

\section{Implications for research}

Paramedics in this study were motivated individuals and demonstrated clear commitment to their professional role and patient care. Their enthusiasm to extend skills through participation in research confirms the feasibility of undertaking research in these settings to inform new practice. $^{30}$

\section{Strengths and limitations}

Paramedics in this study were a self-selected group of individuals willing to share their views in focus group discussion and from one small area of South Wales. We do not know the experience of paramedics who participated in RAPID but who did not attend the focus groups. Although we held focus groups on different days and at different times, shift systems and rota working may have prevented more people from attending. Nor do we know their views on extending the paramedic role into undertaking clinical procedures such as FICB, including any concerns on system issues (eg, indemnity and contracts) which focus group respondents did not highlight. It is possible that the levels of motivation and attitudes towards skills development reported by those who took part in group discussions may not be typical of all paramedics. While we do not know yet whether paramedics generally will be willing to administer FICB, that does not contradict our findings that paramedics are able successfully to learn and practice the procedure.

A strength of this study is the broad perspective which our multidisciplinary team brought, to the analysis in particular and the whole study generally. Our specialist input included paramedics, anaesthetists, patients, carers, methodologists and ambulance service managers. Our qualitative analysis team involved two researchers, a patient and a paramedic. Additionally, our qualitative work within this feasibility study enabled us to explore implementation issues to inform our ongoing research. ${ }^{14}$ We have amended our proposed paramedic training procedures to include opportunities for more familiarity with drugs packs, more scenario-based training and regular refresher sessions.

\section{Future research}

This paper reports results which are part of the RAPID feasibility study. ${ }^{13}$ Having demonstrated that a randomised trial of FICB is feasible and met our predefined progression criteria, ${ }^{16}$ we shall prepare a proposal for a fully powered multicentre randomised controlled trial. This will provide an opportunity to evaluate whether FICB is clinically effective for patients and is cost effective for the
NHS. This reflects the wider NHS strategy to provide the right care to the patient and improve the efficiency and effectiveness of patient journeys to and through hospital.

Author affiliations

${ }^{1}$ Medical School, Swansea University, Swansea, UK

${ }^{2}$ Public contributor, c/o Swansea University, Swansea, UK

${ }^{3}$ Abertawe Bro Morgannwg University Health Board, Port Talbot, UK

${ }^{4}$ Abertawe Bro-Morgannwg University Health Board, Cardiff, UK

${ }^{5}$ The Welsh Ambulance Services NHS Trust, Swansea, UK

${ }^{6}$ School of Medicine, Cardiff University, Cardiff, UK

${ }^{7}$ Pre-hospital Emergency Research Unit, Welsh Ambulance Services NHS Trust, Cardiff, UK

Acknowledgements We thank all the paramedics who volunteered to take part in RAPID, and gave their own time to undertake training and attend focus groups; and the patients and their families who took part in interviews about their experiences. We thank the members of the Trial Steering Committee: Chris Foy (Chair and Statistician, Gloucestershire Hospitals NHS R\&D Service); Tim Coats (Professor of Emergency Medicine, University of Leicester); Matt Costa (Professor of Orthopaedic Trauma Surgery, Oxford University and John Radcliffe Hospital); Philip Bell (Lay Member) and Graham McLelland (Research Paramedic, North East Ambulance Service). We also thank Emma Baker and Carys Evans project administrators, for their hard work throughout this feasibility study.

Contributors $B A E$ drafted the manuscript with editorial input from all authors- $A B$, JB, GF, SF, KG, SJ, LK, AK, ML, IP, NR, ACS, ITR, AW and HS. BAE led qualitative analysis with JB, SJ and LK. The research idea was conceived and developed by NR, IP and SF, with methodological advice from GF, HS and ITR. All authors read and approved the final manuscript.

Funding The Health and Care Research Wales, part of the Welsh Government, has funded RAPID through their Research for Patient and Public Benefit (RfPPB) stream (1003).

Competing interests IP is Director of Trauma Simulation, which produced the bespoke mannequin used in training. The other authors declare that they have no competing interests.

Patient consent for publication Not required.

Ethics approval Wales Research Ethics Committee 6 (reference 15/WA/0439).

Provenance and peer review Not commissioned; externally peer reviewed.

Data sharing statement There are no additional unpublished data from this study.

Open access This is an open access article distributed in accordance with the Creative Commons Attribution Non Commercial (CC BY-NC 4.0) license, which permits others to distribute, remix, adapt, build upon this work non-commercially, and license their derivative works on different terms, provided the original work is properly cited, appropriate credit is given, any changes made indicated, and the use is non-commercial. See: http://creativecommons.org/licenses/by-nc/4.0/.

\section{REFERENCES}

1. Evans R, McGovern R, Birch J, et al. Which extended paramedic skills are making an impact in emergency care and can be related to the UK paramedic system? A systematic review of the literature. Emerg Med J 2014;31:594-603.

2. England NHS, Safer F. Better: good practice in delivering urgent and emergency care. A Guide for local health and social care communities. 2015 https://improvement.nhs.uk/documents/701/ Safer-Faster-Better.pdf (Accessed 5th Jan 2017).

3. Bigham BL, Kennedy SM, Drennan I, et al. Expanding paramedic scope of practice in the community: a systematic review of the literature. Prehosp Emerg Care 2013;17:361-72.

4. Friedman SM, Mendelson DA. Epidemiology of fragility fractures. Clin Geriatr Med 2014;30:175-81.

5. National Institute for Health and Care Excellence. Hip Fracture in adults Quality Standard [QS16]. 2012 http://www.nice.org.uk/ guidance/qs16 (Accessed 5th Jan 2017).

6. Callear J, Shah K. Analgesia in hip fractures. Do fascia-iliac blocks make any difference? BMJ Qual Improv Rep 2016;5:u210130.w4147.

7. Simunovic N, Devereaux PJ, Sprague S, et al. Effect of early surgery after hip fracture on mortality and complications: systematic review and meta-analysis. CMAJ 2010;182:1609-16. 
8. Holdgate A, Shepherd SA, Huckson S. Patterns of analgesia for fractured neck of femur in Australian emergency departments. Emerg Med Australas 2010;22:3-8.

9. Cowan R, Lim JH, Ong T, et al. The challenges of anaesthesia and pain relief in hip fracture care. Drugs Aging 2017;34:1-11.

10. Chaudet A, Bouhours G, Rineau E, et al. Impact of preoperative continuous femoral blockades on morphine consumption and morphine side effects in hip-fracture patients: a randomized, placebo-controlled study. Anaesth Crit Care Pain Med 2016;35:37-43.

11. Simpson PM, Bendall JC, Tiedemann A, et al. Provision of out-ofhospital analgesia to older fallers with suspected fractures: above par, but opportunities for improvement exist. Acad Emerg Med 2013;20:761-8.

12. McRae PJ, Bendall JC, Madigan V, et al. Paramedic-performed fascia iliaca compartment block for femoral fractures: a controlled trial. J Emerg Med 2015;48:581-9.

13. Bulger JK, Brown A, Evans BA, et al. Rapid analgesia for prehospital hip disruption (RAPID): protocol for feasibility study of randomised controlled trial. Pilot Feasibility Stud 2017;3.

14. O'Cathain A, Hoddinott P, Lewin S, et al. Maximising the impact of qualitative research in feasibility studies for randomised controlled trials: guidance for researchers. Pilot Feasibility Stud 2015;1:32.

15. Clement C, Edwards SL, Rapport F, et al. Exploring qualitative methods reported in registered trials and their yields (EQUITY): systematic review. Trials 2018;19:589.

16. Bulger JK, Brown A, Evans BA, et al. Rapid analgesia for prehospital hip disruption (RAPID): protocol for feasibility study of randomised controlled trial. Pilot Feasibility Stud 2017;3:8.

17. Keen L, Bulger JK, Rees N, et al. Use of scratchcards for allocation concealment in a prehospital randomised controlled trial. Emergency Medicine Journal 2018;359:emermed-2018-207881-710.

18 . http://e-safe-anaesthesia.org/e_library/09/Fascia_lliaca compartment block_TOTW 193 2010.pdf (Accessed 1st Nov 2018)
19. Association of Anaesthetists of Great Britain and Ireland. Fascia iliaca blocks and non-physician practitioners. London: AAGNI, 2013.

20. Wolff M, Wagner MJ, Poznanski S, et al. Not another boring lecture: engaging learners with active learning techniques. $J$ Emerg Med 2015;48:85-93.

21. Ziebland S, McPherson A. Making sense of qualitative data analysis: an introduction with illustrations from DIPEx (personal experiences of health and illness). Med Educ 2006;40:405-14.

22. Evans $B A$, Bedson $E$, Bell $P$, et al. Involving service users in trials: developing a standard operating procedure. Trials 2013;14:219.

23. Mercuri M, Mondoux SE. Tackling the demand for emergency department services: there are no silver bullets. Emerg Med $J$ 2018;35:3-4.

24. Ball L. Setting the scene for the paramedic in primary care: a review of the literature. Emerg Med J 2005;22:896-900.

25. Noble AJ, Snape D, Goodacre S, et al. Qualitative study of paramedics' experiences of managing seizures: a national perspective from England. BMJ Open 2016;6:e014022.

26. Sherratt FC, Snape D, Goodacre S, et al. Paramedics' views on their seizure management learning needs: a qualitative study in England. BMJ Open 2017;7:e014024.

27. Lees D, Harrison WD, Ankers T, et al. Fascia iliaca compartment block for hip fractures: experience of integrating a new protocol across two hospital sites. Eur J Emerg Med 2016;23:12-18.

28. Daniels AH, Daiello LA, Lareau CR, et al. Preoperative cognitive impairment and psychological distress in hospitalized elderly hip fracture patients. Am J Orthop 2014;43:E146-52.

29. National Institute for Health and Care Excellence (NICE). The management of hip fracture in adults. 2017 https://www.nice.org.uk/ Guidance/CG124 (Accessed 25th Jan 2018).

30. Craig P, Dieppe P, Macintyre S, et al. Developing and evaluating complex interventions: the new medical research council guidance. BMJ 2008;337:a1655. 